\title{
Systematic extraction of diagnostic data items for common high-risk pregnancies using Delphi technique
}

\author{
Type of article: conference abstract
}

\author{
Kolsoum Deldar1, Seyed Mahmood Tara2*, Majid Jangi1 \\ $1 \mathrm{PhD}$ candidate of Medical Informatics, Student Research Committee, Department of \\ Medical Informatics, Faculty of Medicine, Mashhad University of Medical Sciences, \\ Mashhad, Iran. \\ 2Assistant professor, Department of Medical Informatics, Faculty of Medicine, Mashhad \\ University of Medical Sciences, Mashhad, Iran. \\ *Email: Taram@mums.ac.ir,
}

\begin{abstract}
Introduction: The quality of clinical decisions being made every day by on-call physicians are totally based on the quality of medical information they receive during telephone consultations with residents. Some basic factors such as the right selection of medical items, type and format, and also the volume of such information may highly affect the quality of remote consultations. Therefore, developing a trusted standard model for such clinical communication seems vital. In this research, we used Delphi technique to develop a set of information items in form of clinical decision archetypes to standardize teleconsultation in high-risk pregnancies.

Methods: A multi-stage cross-sectional study was conducted to exploit the diagnostic items for the most common high-risk pregnancies in three obstetrics and gynecology department of educational hospitals, Mashhad, Iran.

Results: Our study revealed eclampsia/preeclampsia, hemorrhage, PROM, pre-term and post-term delivery as the most common high-risk pregnancies in the hospitals being studied. 189 clinicallyimportant items were extracted from scientific references and then hand-filtered to 128 items by the participating gynecology and obstetrics experts. The final items were categorized into five classes including general information, chief complaint / current problem, medical history, clinical examination, and paraclinic tests.

Conclusion: In this study, a set of clinical decision archetype was developed to improve the decisions being made in high risk pregnancies.

Keywords: High-risk pregnancy, medical consultation, remote consultation
\end{abstract}

\section{Declaration of conflicts}

This abstract is selected from the First International Congress of Diseases and Health Outcomes Registry and First National Congress of Medical Informatics, 14-17 February 2017, Mashhad, Iran

\section{Authors' biography}

No biography.

\section{References}

No references. 